Revista de Dialectología y Tradiciones Populares, vol. LXXIII, n. ${ }^{\circ}$ 2, pp. 387-406, julio-diciembre 2018 ,

ISSN: 0034-7981, eISSN: 1988-8457,

https://doi.org/10.3989/rdtp.2018.02.006

\title{
El discurso sobre la gestión intercultural de la diversidad en Barcelona*
}

\section{The Discourse on the Intercultural Management of the Diversity in Barcelona}

\author{
Diana Mata Codesal ${ }^{1}$ \\ Universidad Pompeu Fabra
}

\section{RESUMEN}

Tomando como ejemplo los planes de interculturalidad y gestión de la inmigración de la ciudad de Barcelona, este artículo analiza críticamente tres ideas que se toman como axiomáticas respecto al ámbito de las migraciones y la gestión de la diversidad en esos documentos políticos, y de qué manera estos utilizan, adaptan u omiten desarrollos académicos al respecto. El artículo analiza, en primer lugar, la afirmación de que ha habido un aumento de la diversidad en la ciudad como consecuencia de las migraciones internacionales. En segundo lugar, el hecho de que la diversidad cultural plantea una situación nueva que hace falta gestionar. Por último, el artículo analiza la afirmación de que la interacción entre personas socioculturalmente distintas, elemento este que se encuentra en la base de los distintos planteamientos denominados interculturales de gestión de la diversidad cultural, es buena y tiene consecuencias sociales positivas.

Palabras clave: Migraciones; Diversidad; Gestión; Interculturalidad; Interacción; Barcelona.

\section{SUMMARY}

Taking the example of interculturality and immigration management plans for the city of Barcelona, this article critically analyses three ideas considered axiomatic regarding migration and the management of diversity in these political documents, and explores how academic developments are used, adapted or omitted in the matter. First, the article analyses the claim that diversity in the city has increased as a consequence of international migration. Secondly, it examines the notion that cultural diversity has thrown up a new situation that requires management. And

* La investigación que recoge este trabajo ha sido financiada por una ayuda postdoctoral Beatriu de Pinós/Marie Curie Cofund con referencia AGAUR 2014 BP B 00262. Como con todos los trabajos antropológicos, este texto no hubiera sido posible sin la colaboración de muchas personas de dentro y fuera del mundo académico. Quiero agradecer a Concepción Maiztegi por sus comentarios a una versión previa de este trabajo, así como los de las personas revisoras anónimas, que han ayudado a afinar el resultado final. Gracias también a todas las personas de la lista de distribución del Grupo de Investigación sobre Antropología del Conflicto Urbano y a su alma mater, Manuel Delgado, por la increíble generosidad intelectual que profesan, algo por desgracia poco común en los entornos académicos en estos tiempo. En especial quisiera agradecer a todas las integrantes de la Asociación de Vecinos y Vecinas del Carmelo por seguir y por los tiempos compartidos.

${ }^{1}$ Correo electrónico: d.mata.codesal@gmail.com. ORCID iD: <https://orcid.org/0000-0002-1438-7133>. 
finally, it considers the claim that interaction among socio culturally different persons-an element underlying various so-called 'intercultural' approaches to managing cultural diversity-is good and has socially positive consequences.

Keywords: Migration; Diversity; Management; Interculturality; Interaction; Barcelona.

\section{INTRODUCCIÓN}

Como establecía el ex alcalde de Barcelona Joan Clos en la introducción del primer Plan Municipal para la Interculturalidad en Barcelona de 1997 "Las ciudades europeas son cada vez más multiétnicas [...] Barcelona es también la suma de muchas tradiciones y culturas, el resultado del enriquecimiento que supone la diversidad cultural [...]" (Ayuntamiento de Barcelona 1997: 5). En palabras de este mismo alcalde, recogidas esta vez en la introducción del Plan Municipal de Inmigración de la ciudad de 2002, en la ciudad de Barcelona desde finales del siglo XX se percibe que la "oleada de inmigración es muy diferente a las del siglo XX porque nos aporta y nos acerca culturas que son muy distantes desde todos los puntos de vista: lingüístico, religioso, de hábitos alimenticios, higiénicos. O sea, culturas manifiestamente diferentes, alejadas" (Ayuntamiento de Barcelona 2002: 71). Unos años después el Plan de Trabajo de Inmigración de Barcelona para los años 2008-2011 establece que "[e]l fuerte aumento de la diversidad sociocultural de la ciudad es a la vez fuente de oportunidades, pero también de mayores complejidades, y que depende de cómo la interpretemos y gestionemos para que prevalezcan unos factores u otros" (Ayuntamiento de Barcelona 2008: 71). La diversidad cultural, y en concreto "la gestión de la convivencia en la diversidad" como establece Jordi Hereu, otro ex alcalde de Barcelona, en el prólogo del Plan Barcelona Interculturalidad de 2010 (Ayuntamiento de Barcelona 2010: 5), deviene un reto así como una oportunidad para la convivencia y la cohesión social en la ciudad. Xavier Trias, alcalde de Barcelona entre 2011 y 2015, coincide con sus antecesores al establecer en el Plan de Trabajo de Inmigración 20122015 que las autoridades tienen un papel importante que jugar en la gestión de esta nueva realidad «estableciendo condiciones que fomenten la interacción positiva entre personas de distintas culturas para generar diálogo y conocimiento mutuo" (Ayuntamiento de Barcelona 2012: 3) lo que sin lugar a dudas repercutirá, entre otros aspectos, en una reducción de las actitudes xenófobas y una mejora de la cohesión social ya que, como recogía el citado Plan Municipal de Inmigración de Barcelona de 2002, "si la población que llega a la ciudad pertenece además, a otros dominios culturales y tiene hábitos y pautas que no son propios de la sociedad de acogida, la presión de los movimientos migratorios puede desembocar en conflicto y pueden aparecer prejuicios y brotes de xenofobia" (Ayuntamiento de Barcelona 2002: 77) ${ }^{2}$.

El párrafo anterior, que combina citas procedentes de los planes municipales en materia de integración y gestión de la diversidad aprobados en la ciudad de Barcelo-

\footnotetext{
${ }^{2}$ La inversión de la carga de la responsabilidad es una falacia peligrosa y extendida como ya mostró Hannah Arendt (2003) en su ensayo sobre la banalidad del mal por cuanto de este mecanismo de culpabilización de la víctima implícitamente se deduce que es la presencia de inmigrantes la causa de la xenofobia.
} 
na en los últimos veinte años entre 1997 y 2017³, recoge tres ideas respecto al ámbito de las migraciones y la gestión de la diversidad en esa ciudad que se repiten frecuentemente en documentos políticos. A saber, 1) que ha habido un aumento de la diversidad en la ciudad como consecuencia de las migraciones internacionales; 2) que la diversidad cultural plantea una situación nueva que hace falta gestionar; y 3) que la interacción entre personas socioculturalmente distintas (elemento este que se encuentra en la base de los distintos planteamientos denominados interculturales de gestión de la diversidad cultural) es buena y tiene consecuencias sociales positivas.

En este texto analizo esas tres afirmaciones que son habitualmente tomadas como axiomáticas en el discurso político, y cómo estas interpelan, apropian, tergiversan o ignoran los debates académicos relacionados, en especial las aportaciones conceptuales relativas a la cultura y la diversidad hechas desde la antropología. Para ello utilizo el caso de Barcelona ilustrándolo con datos etnográficos generados en uno de sus barrios periféricos, el barrio del Carmelo ${ }^{4}$. Barcelona es un caso de estudio especialmente interesante ya que esta ciudad ha sido tanto canal de entrada como espacio generador de ideas y aplicaciones concretas en el ámbito de la integración de las personas migradas y la denominada gestión de la diversidad. Muchos de estos enfoques se han extendido posteriormente hacia otras ciudades españolas. Como veremos, la estrategia antirrumores que forma uno de los pilares del actual Programa de Interculturalidad de la ciudad continúa siendo el modelo a seguir para la implementación de la estrategia en muchas otras ciudades españolas 5

Antes de continuar se hace necesaria una reflexión dada la confusión conceptual en torno a las nociones ampliamente utilizadas de interculturalidad e interculturalismo. Giménez (2003) provee una temprana aclaración conceptual para el caso concreto de la educación al diferenciar claramente entre el ámbito de la realidad social (lo que es y en cierta manera siempre ha sido) y el de la ideología política (lo que se cree que debería ser) ${ }^{6}$. Por un lado, la interculturalidad es el contacto entre personas diferentes bien porque profesan diferente religión, hablan lenguas distintas o, entre otros elementos, viven de acuerdo a cosmovisiones diferentes. Por el contrario, el interculturalismo es la ideología política que entiende que la diversidad cultural es buena y que hace falta intervención pública para asegurar los principios de igualdad, diferencia e interacción positiva. Esta reflexión se ampliará en la sección 4.3 al cuestionar el tercer axioma, el de la interacción, ya que la distinción interculturalidad e interculturalismo en ocasiones se difumina, lo que tiene consecuencias importantes.

Este artículo se estructura en cinco apartados. En la siguiente sección se presentan de manera resumida los planes sobre migraciones e interculturalidad aprobados

\footnotetext{
${ }^{3}$ Los planes fueron aprobados por tres alcaldes diferentes: Joan Clos y Jordi Hereu (ambos del PSC-Partido Socialista de Catalunya), así como Xavier Trias (CIU-Convergencia y Unión).

${ }^{4}$ Utilizo la acepción castellana Carmelo en lugar de la oficial en catalán, El Carmel, porque es la que la mayoría de quienes habitan en el barrio utilizan habitualmente.

${ }^{5}$ Este efecto transmisor de las autoridades de Barcelona no se limita al ámbito de la gestión de la inmigración y la integración de las personas migradas, como claramente muestra la polémica Ordenanza de Civismo aprobada en la ciudad en 2006 y que ha sido el modelo para regulaciones similares en muchas otras ciudades españolas (Ayuntamiento de Barcelona 2006).

${ }^{6}$ Los fundamentos de la educación intercultural, que se inicia tempranamente en América Latina con la educación indígena, en general no se han trasladado bien al campo de la ciencia política europea lo cual ha generado confusión conceptual.
} 
en Barcelona. El apartado 3 recoge de forma muy breve la parte empírica del trabajo. Tal brevedad se debe al hecho de que este texto utiliza de manera ilustrativa dinámicas sociales de un barrio periférico de Barcelona para realizar un análisis crítico-antropológico del modo en el que los discursos académicos-antropológicos son apropiados por los discursos políticos en el campo de la gestión de la diversidad. Este artículo por tanto es más un texto de revisión documental que un texto etnográfico al uso. El cuarto apartado analiza las tres ideas fundamentales que subyacen a los mencionados planes y a ciertas aplicaciones del interculturalismo. El artículo termina con algunas ideas a modo de conclusión.

\section{BARCELONA, LA ESTRATEGIA INTERCULTURAL}

Es a partir de finales de siglo XX cuando en España, tanto a nivel municipal, autonómico como estatal, la preocupación política pasa de centrarse únicamente en la cuestión de la entrada y recepción de inmigrantes y se comienza a pensar en los efectos a medio y largo plazo de la presencia de esta población, empezándose a hablar de la integración de los inmigrantes. Dado que el análisis pormenorizado de cada uno de los planes excede el objetivo de este artículo, la Tabla 1 recoge los principales planes de inmigración de la ciudad de Barcelona, así como los planes realizados a nivel estatal y autonómico en Cataluña con el único fin de contextualizar cronológicamente los desarrollos ocurridos en los tres niveles de gobierno.

El desarrollo del modelo intercultural para la integración en el caso de Barcelona está marcado por tres documentos claves: el temprano Un Plan Municipal para la Interculturalidad de 1997, el ambicioso Plan Bcn Interculturalidad de 2010, y el Programa Bcn Interculturalidad en vigor desde 2016. El Plan Municipal para la Interculturalidad aprobado en 1997 ha de entenderse más como una declaración de principios y de posicionamiento político que como un plan operativo. Este plan es importante porque muchas de las ideas actuales sobre interculturalidad en la ciudad estaban ya presentes en él. Su objeto de interés eran las "personas inmigrantes extranjeras y las minorías étnicas", aunque se reconocía que su presencia era baja en la ciudad y se hacía además la distinción entre extranjeros provenientes de países del Sur y países del Norte (Ayuntamiento de Barcelona 1997: 9). Los sujetos de aplicación de los cinco ejes programáticos del plan eran "los organismos y servicios municipales [habiéndose] de compartir con el tejido asociativo y con toda la ciudadanía" (Ibid. 1997: 13). El plan se asienta sobre una visión de la diversidad cultural como fuente de riqueza e incorpora de manera clara la retórica intercultural como opuesta al asimilacionismo. Es claramente progresista por cuanto establece medidas de tipo material-estructural que sin embargo no se llegaron a aplicar, como por ejemplo un acceso a los servicios municipales basado únicamente en la distinción entre transeúnte y residente en la ciudad y el establecimiento de mecanismos justificadores de residencia alternativos si el padrón no fuese posible (1997: 14). El plan enfatiza la no discriminación, el acceso adecuado a la información para las personas migradas, la promoción de una imagen positiva de las mismas y la reducción del prejuicio (algo que años después se convertirá en un punto central del Plan Barcelona Interculturalidad 2010 con la estrategia antirrumores), así como la promoción del asociacionismo. Es en general un plan ambicioso pero ambiguo en sus ejes programáticos. 


\begin{tabular}{|c|c|c|c|}
\hline AÑO & BARCELONA & CATALUÑa & ESPAÑA \\
\hline 1993 & & $\begin{array}{l}\text { I Plan Interdepartamental de } \\
\text { Inmigración 1993-2000 }\end{array}$ & \\
\hline 1994 & & & $\begin{array}{l}\text { Plan para la Integración So- } \\
\text { cial de los Inmigrantes (PISI) }\end{array}$ \\
\hline 1997 & $\begin{array}{l}\text { Un Plan Municipal para la } \\
\text { Interculturalidad }\end{array}$ & & \\
\hline 2001 & & $\begin{array}{l}\text { II Plan Interdepartamental de } \\
\text { Inmigración 2001-2004 }\end{array}$ & $\begin{array}{l}\text { Programa Global de Regula- } \\
\text { ción y Coordinación de Extran- } \\
\text { jería e Inmigración (GRECO) }\end{array}$ \\
\hline 2002 & $\begin{array}{l}\text { PMI-Plan Municipal de Inmi- } \\
\text { gración } 2002\end{array}$ & & \\
\hline 2005 & & $\begin{array}{l}\text { Plan de Ciudadanía e Inmi- } \\
\text { gración 2005-2008 }\end{array}$ & \\
\hline 2007 & & & $\begin{array}{l}\text { Plan Estratégico Ciudadania e } \\
\text { Integración 2007-2010 (PECI) }\end{array}$ \\
\hline 2008 & $\begin{array}{l}\text { Plan de Trabajo de Inmigra- } \\
\text { ción 2008-2011 }\end{array}$ & & \\
\hline 2009 & & $\begin{array}{l}\text { Plan de Ciudadanía e Inmi- } \\
\text { gración 2009-2012 }\end{array}$ & \\
\hline 2010 & Plan Bcn Interculturalidad & & \\
\hline 2011 & & & $\begin{array}{l}\text { Plan Estratégico de Ciudada- } \\
\text { nía e Integración 2011-2014 } \\
\text { (PECI II) }\end{array}$ \\
\hline 2012 & $\begin{array}{l}\text { Plan de Trabajo en Inmigra- } \\
\text { ción 2012-2015 }\end{array}$ & & \\
\hline 2013 & & $\begin{array}{l}\text { Plan de Ciudadania y de las } \\
\text { Migraciones: Horizonte } 2016\end{array}$ & \\
\hline 2016 & $\begin{array}{l}\text { Programa Bcn Intercultu- } \\
\text { ralidad }\end{array}$ & & \\
\hline
\end{tabular}

TABLA 1.-Principales planes y programas de inmigración en el nivel municipal, autonómico y estatal entre 1993 y $2017^{7}$. Fuente: Elaboración propia

Tras el Año Europeo del Diálogo Intercultural (2008), se inicia en 2009 un amplio proceso consultivo con gran número de entidades de la ciudad que culminará en 2010 con la aprobación del Plan Barcelona Interculturalidad. Este plan consolida el

\footnotetext{
${ }^{7}$ El primer plan de integración de los inmigrantes a nivel estatal —PISI, Plan para la Integración Social de los Inmigrantes - se aprueba en 1994. Un año antes en Cataluña, la Generalitat aprueba el I Plan Interdepartamental de Inmigración. En la actualidad a nivel estatal aún no se procedido a la evaluación del PECI II, Plan Estratégico de Ciudadanía e Integración 2011-2014 ni a la elaboración de un nuevo plan (FISI 2016). A nivel autonómico se encuentra vigente el quinto plan aprobado por la Generalitat denominado Plan de Ciudadania y de las Migraciones: Horizonte 2016.
} 
interculturalismo como modelo normativo para la gestión de la diversidad cultural en la ciudad. Los diez ejes programáticos del plan pretenden superar situaciones de mera coexistencia para lograr situaciones de convivencia enfatizando elementos como la lengua, el respeto a las normas de convivencia o la importancia de la educación y el asociacionismo. La interacción personal es el eje pivotante de todo el plan, ya que como establece "[e]ntendemos que la apuesta por la interacción es la mejor garantía para la convivencia y la cohesión social, y también para aprovechar al máximo las oportunidades de la diversidad" (Ayuntamiento de Barcelona 2010: 31). De manera similar al plan de 1997, se asigna a la administración y el tejido social local el papel de crear las condiciones para que las interacciones se den en clave de positiva en un marco de ganancia mutua para todas las partes implicadas al entender la diversidad cultural como un activo para la ciudad. Por primera vez, el plan incorpora un amplio listado de indicadores de seguimiento.

El plan más reciente y ambicioso hasta la fecha es el Programa Barcelona Interculturalidad (2016) que engloba el Plan Barcelona Interculturalidad aprobado en 2010 junto con algunos elementos del Plan de Trabajo en Inmigración 2012-2015 municipal. Este programa sigue en esencia los planteamientos del plan de 2010 que estructura en torno a cuatro grandes áreas o estrategias. En primer lugar, y de manera destacada, la estrategia antirrumores cuya lógica y resultados serán analizados con más detalle en las próximas secciones. El segundo eje es el espacio Avinyó de Lengua y Cultura, surgido en coordinación con el Consorcio por la Normalización Lingüística de la Generalitat de Catalunya y que inició sus actividades dando gran preponderancia a la lengua para pasar a incluir cada vez más temas de diálogo interreligioso y fomentar la visibilidad de manifestaciones artísticas diversas. El tercer pilar lo conforman distintos programas de formación intercultural dirigidos a grupos diferentes de la ciudad. Finalmente, el programa recoge y pone a disposición de las distintas entidades locales un amplio catálogo de recursos clasificados como interculturales generados tanto por las autoridades municipales como por otros agentes de la ciudad.

En la primera mitad de 2017 aún no se había aprobado ningún nuevo plan aunque desde 2016 está en marcha un proceso de evaluación del plan vigente y de elaboración de uno nuevo. En septiembre de 2016 aparece un Informe sobre los Retos y Oportunidades del Programa Bcn Interculturalidad (2016a: 4) donde se plantea explícitamente un cambio paradigmático al "entender las políticas públicas de gestión de la diversidad cultural de manera global y no solo vinculadas a los procesos migratorios como hasta el momento". Según la información disponible en el momento de escritura de este texto hay varios elementos que marcan, al menos sobre el papel, un cambio en el enfoque aplicado. Por un lado se sustituye el énfasis en la interacción positiva por el interés en los entramados relacionales al entender que las relaciones sociales no están exentas de conflictos. En segundo lugar y como respuesta esperable a la evolución temporal del fenómeno migratorio, se incorpora un interés por la realidad de las personas de ascendencia migrante. En tercer lugar se busca incorporar explícitamente las cuestiones que atañen al pueblo gitano. Y por último, se apuesta por regular aquellos elementos relacionados con la diversidad religiosa. 


\section{DEL CARMELO AL CIELO}

La expresión que titula esta sección, "Del Carmelo al cielo", es una expresión de uso habitual por los habitantes de este barrio que condensa tres elementos: 1) la posición geográfica del barrio, situado en las faldas de dos pequeñas colinas (Turó de la Rovira y Turó del Carmel); 2) una reacción de sus habitantes frente a la fuerte estigmatización espacial que tradicionalmente ha sufrido el barrio y sus habitantes por parte de ciertos sectores de la ciudad de Barcelona; y 3) la preponderancia del uso del castellano, en comparación con el catalán, de los habitantes del barrio, en especial de aquellos que vinieron de otras partes de España y a quienes se considera fundadores del barrio, pero también de sus descendientes cuyo idioma materno sigue siendo el español. El Carmelo es por tanto un barrio situado en la periferia tanto geográfica como social de Barcelona. La periferia social queda clara al comprobar cómo la renta familiar disponible per cápita en el Carmelo en 2015 era casi la mitad que la de la ciudad en su conjunto (Ayuntamiento de Barcelona 2016b). Desde el establecimiento del barrio en su forma actual por migrantes internos con posterioridad a la Guerra Civil, este barrio presenta sistemáticamente un porcentaje más alto de población nacida en otras partes del país que la media de Barcelona. En 2016 el 29,5 \% de la población del barrio había nacido fuera de Cataluña frente al 18,2 \% de Barcelona. Ese mismo año en el barrio vivía un decreciente 18,4 \% de población nacida en otros países frente al 22,5\% para Barcelona en su conjunto (Ayuntamiento de Barcelona 2016c). Comparten espacio geográfico por tanto viejos vecinos venidos de otras partes de España, con nuevos vecinos provenientes de fuera de España.

Esta realidad demográfica se despliega en una realidad espacial precaria, ya que el Carmelo presenta una escasez de espacios públicos de acceso abierto, lo que unido a un tradicional olvido urbanístico por parte de las autoridades, genera espacios donde confluyen muchas personas que además los utilizan de maneras diferentes, en especial en cuanto a sus edades, con una tensión muy fuerte entre jóvenes/adolescentes y personas ancianas. Estas tensiones generacionales son presentadas por ciertos vecinos de edad avanzada como tensiones culturales, de la misma manera que ha sucedido en otros espacios de la ciudad (para el caso de Ciutat Vella ver Aramburu 2002). Para hacer frente a estas tensiones entre viejos y nuevos vecinos (con tales adjetivos haciendo referencia tanto a la edad de las personas como al tiempo de residencia en el barrio) desde la administración local se ha promovido una gestión intercultural de la diversidad en algunos espacios públicos del barrio. Es importante mencionar que el antiguo servicio municipal denominado Nouvinguts ('Recién Llegados') destinado a ofrecer asesoramiento e información básica a personas migradas, ha pasado a convertirse en el Servicio de Convivencia. Este servicio ha sido el espacio coordinador de las distintas iniciativas institucionales de gestión de los conflictos que han tenido lugar en algunos espacios públicos del barrio entre los que destaca la Mesa de Convivencia de Plaza Pastrana y Alrededores que reúne de manera periódica a entidades del barrio y vecinos y vecinas de la denominada plaza para tratar los "problemas de convivencia" en ese espacio. Muchas de las iniciativas de mejora promovidas se limitan a acciones de embellecimiento del paisaje urbano como la realización de grafitis en persianas colindantes, a una mayor presencia de cuerpos policiales y equipos de limpieza municipales, así como a otras acciones de disuasión 
suave sobre ciertos grupos, en especial de jóvenes y adolescentes, por parte de educadores de calle y otros equipos técnicos del ayuntamiento. Desde las autoridades se ha impuesto por tanto un enfoque de regulación de la interacción en la línea del tutelaje pedagógico que se discutirá más adelante, y que persigue mostrar las maneras correctas de estar en el espacio público en aras de una convivencia desconflictivizada. El denominado Servicio de Convivencia carece de los recursos y el poder para implementar cambios urbanísticos de calado que dotarían al barrio de más y mejores espacios públicos, lo que sin duda aliviaría la tensión de uso de espacios como plaza Pastrana.

El diseño metodológico de la investigación se compone de veinte entrevistas semiestructuradas con personas que trabajan en alguna asociación del barrio o viven en él (tanto vecino/as recientes como de toda la vida), la participación en dos organizaciones del barrio (una tradicional, la Asociación de Vecinos y Vecinas del Carmelo, y otra de nueva aparición, un grupo de consumo) durante todo el año 2016, observaciones no intrusivas (Pétonnet 1982) en "La Pastrana" (como se conoce coloquialmente a la plaza Pastrana) durante dos meses y el desarrollo de tres fotopaseos sobre diversidad y espacio público en el barrio. La participación en la Asociación de Vecinos/as permitió un acceso privilegiado tanto a las problemáticas del barrio como a la respuesta institucional ya que esta asociación actúa como interlocutor legítimo ante las autoridades locales. Las observaciones no intrusivas se llevaron a cabo en la plaza Pastrana, eje neurálgico del barrio, durante los meses de mayo y junio de 2016 en distintas franjas horarias. Esta plaza es una plaza dura, reconstruida a raíz de la llegada del metro al barrio en $2010^{8}$, que presenta gran intensidad de uso, como espacio para descansar, zona de juego para niños y niñas de distintas edades, así como área de paso dada la salida del metro. Las observaciones se centraron en los usos, la distribución espacial de las personas en la plaza, así como en las interacciones que se daban entre distintas personas.

\section{AXIOMAS EN LA GESTIÓN INTERCULTURAL DE LA DIVERSIDAD EN BARCELONA}

Los extractos de documentos políticos redactados a nivel municipal en Barcelona en los últimos veinte años recogidos al inicio de este artículo, aunque elaborados bajo mandatos de diferente signo político, descansan todos ellos en un número reducido de presupuestos comunes. Tales pilares se encuentran habitualmente fuera de toda crítica al presentarse bajo la forma de axiomas, es decir de elementos no discutibles. Para tal transformación, los discursos políticos recurren de manera simplificada a desarrollos supuestamente académicos. Es por tanto necesario analizar cómo los discursos políticos hacen uso de los discursos académicos. Como se verá a continuación para el caso de las tres afirmaciones centrales de tales planes, la complejidad con-

\footnotetext{
${ }^{8}$ Las obras del metro se prolongaron durante muchos más años de los previstos debido al denominado socavón del Carmel ocurrido en enero de 2005. El hundimiento debido a la falta de estudios previos de uno de los túneles afectó a gran número de vecinos/as del barrio que fueron desalojados de sus casas. Tras el desastre se declaró la zona Área Extraordinaria de Rehabilitación Integral (AERI) lo que implicó fuertes mejoras urbanísticas en el barrio (García 2005).
} 
ceptual, y sobre todo, los elementos más críticos de las elaboraciones antropológicas, se difuminan en el traslado que se hace de los mismos al ámbito de las políticas públicas.

\subsection{Vivimos EN CIUDAdES QUE SON MÁS DIVERSAS QUE NUNCA COMO CONSECUENCIA DEL AUMENTO DE LOS FLUJOS MIGRATORIOS}

Muchos trabajos sobre migraciones, gestión de la diversidad e interculturalidad empiezan con la afirmación de que las ciudades son hoy en día más diversas que nunca. Tal afirmación aparece en muchas ocasiones como una realidad sin necesidad de explicación. En otros casos se alude a la magnitud sin precedentes o a la composición diferente de los flujos migratorios actuales como elemento causante, y por tanto explicativo, de tal diversidad urbana. El informe anual de 2015 de la Organización Internacional de las Migraciones Los migrantes y las ciudades: Nuevas colaboraciones para gestionar la movilidad es una cita recurrente al respecto (OIM 2015), así como la utilización del término superdiversidad (Vertovec 2007) para definir la creciente multiplicidad de orígenes y la complejidad de las trayectorias migratorias que encontramos especialmente en las grandes metrópolis.

Sin embargo, afirmar que las ciudades son en la actualidad más diversas que nunca es entender la diversidad de manera exógena a lo urbano, cuando en realidad 1) la diversidad es constituyente de lo urbano, y a la vez 2) lo urbano pone en marcha procesos de diferenciación que operan sobre categorías diferentes dependiendo del tiempo y el lugar concreto. Una vecina entrevistada del Carmelo lo tenía claro al ser preguntada por la diversidad en el barrio:

- ¿Crees que el Carmelo es hoy más diverso que hace treinta años?

-Entonces no hablábamos de diversidad. Solo de diferencia. Pero todo depende de en lo que te enfoques. Al final del día, todos somos diferentes (Vecina de 50 años de toda la vida del Carmelo con experiencia en otras partes de España, entrevista personal, 15 abr. 2016).

Suponer que las ciudades en el pasado tenían menos diversidad porque tenían menos inmigrantes 9 es la imposición de códigos de sentido y clasificatorios actuales, dado que la diferencia socialmente significativa no siempre ha estado sustentada en las características que en la actualidad se consideran relevantes. La diversidad, es decir la copresencia de diferentes, no es un elemento resultante de la movilidad per se, sino de procesos de diferenciación social que en la actualidad toman características asociadas o que de cierta manera se vinculan a (ciertos tipos de) la movilidad internacional. Haber nacido en otro país, poseer otra nacionalidad, hablar otro idioma o tener cierta tonalidad de piel o ciertos rasgos fenotípicos, no son diferencias que tengan que ser en todo contexto necesariamente significativas, sino que son construidas como tal en procesos de diferenciación social, y que se utilizan a posteriori como relevantes para establecer distinciones.

\footnotetext{
9 Esta afirmación es también discutible porque la definición de inmigrante tampoco es una categoría que se pueda descontextualizar de tiempos, espacios y relaciones de poder concretas.
} 
Para operar la diferenciación social es necesario activar mecanismos que conviertan elementos o atributos inicialmente indiferenciados en el material sobre el que efectuar la diferenciación. Es decir, como establece Jean Pouillon (1998: 198), "No clasificamos porque hay cosas que clasificar, sino que al clasificar identificamos elementos para hacerlo", lo que en el caso concreto del racismo cristalizaría en la declaración de Eugenia Ramírez (2011: 458) de que "hay razas porque hay racismo". Los procesos de diferenciación en muchas ocasiones no se limitan a diferenciar, sino que habitualmente llevan a clasificaciones jerárquicas ${ }^{10}$. La movilidad geográfica aparenta ser un criterio fundamental en los procesos de clasificación urbana ya que ciertas migraciones internacionales aparecen en la actualidad como fuente y origen de la creciente diversidad en la ciudad. Como establece Lavanchy, Gajardo y Dervin (2011) el Consejo de Europa se apropió en la década de 1970 del concepto de interculturalidad, un término que venía del terreno de la educación, la comunicación y la psicología, como una medida para gestionar la diversidad cultural traída por los inmigrantes de las excolonias a Europa en un contexto de "emergencia de nuevas definiciones de la otredad, que se designaban como vehículos potenciales de problemas sociales, y que demandaban por tanto medidas de canalización y neutralización de los supuestos riesgos que encarnaban" (Lavanchy, Gajardo y Dervin 2011: 3).

Si prestamos atención al inmigrante como objeto de los estudios sobre diversidad cultural e inmigración y de las políticas de integración, veremos cómo la movilidad no es tan siquiera el hecho definitorio único en la definición de inmigrante (Delgado 2003; Mata Codesal 2016). Como ya varios autores han puesto de manifiesto, el inmigrante no es una categoría únicamente administrativa sino más bien un operador cognitivo que delimita los límites del nosotros (Delgado 2003; Lurbe y Santamaría 2007; Mata Codesal 2016). Un nosotros como categoría que se define en negativo, pero que además es cambiante y por supuesto sujeta a discusión.

En el caso de Barcelona y muy especialmente para el barrio del Carmelo la diferencia relevante ha ido cambiando. Como señalaba Aramburu, en las décadas de 1970 y 1980 los estudios y la preocupación política se centraban en la relación entre las personas venidas de otras partes de España (los denominados charnegos) y la catalanidad o la identidad catalana (Aramburu 2016). Este grupo de interés y objeto de preocupación social y política desapareció casi en su totalidad del mapa político y de investigación en las siguientes décadas ${ }^{11}$, sustituido sin mayor problema por aquellas personas venidas de (ciertos) otros países (Aramburu 2017). En el caso concreto de Barcelona, la oposición catalán-charnego fue central en los procesos de diferenciación posteriores a las migraciones desde otras partes del Estado español, en especial durante las décadas centrales del siglo XX (Clua 2011; Aramburu 2016). La palabra charnego o xarnego ha sufrido modificaciones importantes en su aplicación, desde el que pareciera ser su primer uso para denominar en el siglo XIX a descendientes de matrimonios mixtos entre hombres franceses y mujeres catalanas, hasta el uso que se extendió en las décadas de 1960 y 1970, y que nos interesa aquí, para denominar

\footnotetext{
${ }^{10}$ Podría darse el caso de que ciertos atributos sí fueran percibidos como diferentes pero que no se encontraran imbuidos de significación relevante para los procesos de estructuración social. Es decir, que no fueran elementos con incidencia en los procesos de estructuración social.

${ }^{11}$ La coyuntura política en Cataluña desde 2011 parece haber vuelto a poner en el punto de mira político-social el "problema charnego" (Vassallo 2018).
} 
a los no catalanes venidos de otras partes de España para trabajar en la ciudad (Clua 2011: 67). Aunque el paso del tiempo ha diluido la oposición y esta ha quedado ensombrecida por la nueva alteridad amenazante que opera sobre la figura del inmigrante (quien a manera de elipsis se equipara a personas provenientes del Sur global o las antiguas zonas coloniales), esta continúa vigente, lo que explica los esfuerzos por parte de muchos inmigrantes internos y sus descendientes, como los que conforman buena parte de los habitantes del Carmelo, por diferenciarse de los llegados con posterioridad, y por tanto de manifestar lo inadecuado de aplicar la etiqueta inmigrante a quienes provienen del mismo país.

Cabría preguntarse si en el último siglo han cambiado los procesos en los que la diferencia se sustenta. Los mecanismos en sí no han variado pero sí que han recrudecido sus consecuencias para las personas etiquetadas, debido a la aplicación extra de las categorías legales ${ }^{12}$. La desigualdad ha aumentado por cuanto el régimen de control migratorio dificulta la inserción sociolaboral y económica en condiciones dignas, mientras los mecanismos siguen siendo similares a los que operaron sobre los trabajadores murcianos que a principios del siglo XX llegaron a Barcelona, o sobre la gran masa de inmigrantes de otras partes de España, especialmente de Andalucía y Extremadura, que vinieron con posterioridad.

Así pues, las ciudades no son, porque no pueden serlo, más diversas en la actualidad que en el pasado, ya que la diversidad (es decir, la copresencia de diferentes) es la condición misma de su existencia: «una metrópoli no puede estar hecha de otra cosa que de gente de toda clase, llegada de cualquier parte" (Delgado 2003: 9), ya que desde un punto estrictamente demográfico, la heterogenética o falta de capacidad endógena de muchas ciudades para sostenerse demográficamente por sí mismas, da lugar a una necesidad constante de atraer población desde otras áreas (Vidal 1997).

\subsection{LA DIVERSIDAD CULTURAL NECESITA SER GESTIONADA}

Diversidad es en la actualidad un término de uso ubicuo. El N-grama de Google Books $^{13}$ (Gráficos 1 y 2) con las palabras migración y diversidad muestra claramente cómo a finales del siglo XX esta ha superado a aquella en los libros listados por Google escritos tanto en inglés como en español.

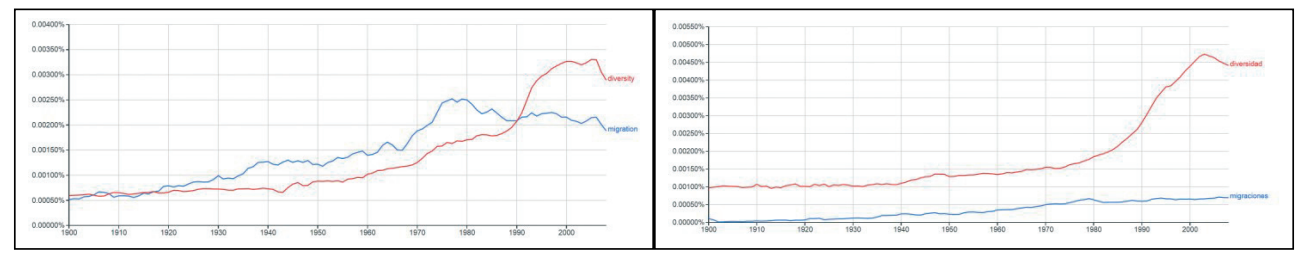

GRÁFICOS 1 Y 2.- N-grama con términos en inglés y n-grama con términos en español. Fuente: Google Books 2017

\footnotetext{
${ }^{12}$ Aunque también se llevaron a cabo algunos procesos de detención y deportación de inmigrantes internos durante el franquismo (Boj 2004).

${ }^{13}$ Este motor de búsqueda en línea representa las frecuencias de palabras en textos reposicionados en Google Books. Lo incluyo aquí solo con fines ilustrativos.
} 
El concepto de diversidad es un ejemplo paradigmático de los diálogos conceptuales existentes entre el mundo académico y el político: un término complejo que es apropiado para su aplicación práctica en sus acepciones más utilitaristas, alejadas de los debates y la complejidad de la misma en las aportaciones antropológicas. Las a priori potencialidades del término quedan a menudo neutralizadas por las apropiaciones y concreciones que se hacen de él (Faist 2009; White 2013; Boccagni 2015). La diversidad ha sido un concepto central para los Management and Organizational Studies, especialmente en Estados Unidos (Boccagni 2015). La gestión de la diversidad (diversity management) es un enfoque dirigido a paliar las desigualdades detectadas en los lugares de trabajo que emerge a mediados de 1980 en ese país (Berrey 2014). En cierta manera, los expertos en recursos humanos van a reformular como gestión de la diversidad las acciones afirmativas, o discriminación positiva, que se habían estado llevando a cabo, y de esta manera "las popularizan con una nueva retórica sobre la maximización del desempeño de las mujeres y las personas de color" (Berrey 2014: 349).

A diferencia de las cacepciones más complejas de la diversidad derivadas de los desarrollos antropológicos, el énfasis actual en la necesidad de gestionar la diversidad señala la adopción del término a partir de los estudios organizacionales. La extensión hacia otras ciencias sociales de esta noción inicialmente relacionada con el mundo de la empresa señala la preocupante tendencia de "la penetración de las lógicas del mercado y sus códigos en los diferentes terrenos institucionales" (Boccagni 2015: 17). No sorprende entonces que en los debates sobre el final del multiculturalismo y los enfoques interculturalismo versus multiculturalismo, la diversidad entendida como "técnica de gestión despolitizada" (Faist 2009: 173) haya encontrado un terreno fértil, lo que está íntimamente relacionado con la crítica más sólida del enfoque de la diversidad hasta la fecha: el hecho de que ignora las desigualdades estructurales (Boccagni 2015). Esta concepción deslavazada es claramente percibida por una trabajadora de una asociación en el Carmelo cuando dice que:

Hay una obsesión por parte de la administración por el tema de la diversidad y la interculturalidad y de programas específicos para la inmigración y ha habido presiones para desarrollar cosas específicas a colectivos [...] Imagínate, con el catálogo este de antirrumores, que en muchos territorios seguro que se puede utilizar, funciona, pero imagínate que yo ahora voy a las cuidadoras, mujeres latinas que cuidan sin contrato de personas mayores, pero por la tipología del territorio, imagínate que yo voy y le ofrezco a hacer una historia de etiquetas, de estereotipos a esa señora que lo que necesita es un contrato laboral para que ella pueda participar en algún asunto (Vecina de 39 años de toda la vida y trabajadora de una asociación del Carmelo, entrevista personal, 13 abr. 2016).

Las visiones celebratorias de la diversidad "a la Benetton" (Lentin y Titley 2008) han oscurecido el inevitable conflicto social en sociedades construidas sobre masivas desigualdades, alienaciones y procesos de desposesión (Caglar y Glick 2015). Tales desigualdades estructuradoras permanecen convenientemente fuera de la vista detrás de los puestos, la música y la comida de los festivales multiculturales y étnicos. En el mejor de los casos, estamos asistiendo a un cambio de marca positivo de la diferencia sin tener que comprometer ni los recursos materiales ni los esfuerzos políticos para cambios sociales profundos (Boccagni 2015: 17).

La cuestión pertinente por tanto no sería qué es la diversidad como qué es lo que los diferentes grupos hacen de ella. El término diversidad ha penetrado en los secto- 
res blancos hegemónicos de clase media de la sociedad para señalar el cuándo, el dónde y el cómo de los grados aceptables de la diferencia. Su versión despolitizada y desconflictivizada ha servido en demasiadas ocasiones como un medio para obviar la desigualdad al folkclorizar partes visibles de la vida de ciertos otros al mismo tiempo que elimina los llamados a la redistribución y el reconocimiento. La promesa del enfoque de la diversidad para analizar las desigualdades cruzadas o intersectadas, y por lo tanto para ayudar a unir las luchas de distintos grupos subordinados, está lejos de ser clara. En España, por ejemplo, casi no hay intentos de unir, teórica o políticamente, las luchas de los migrantes con las de grupos tradicionalmente marginados como los gitanos. La lucha entre grupos explotados y grupos excluidos como Delgado (2016) lo ha etiquetado es un buen ejemplo de las promesas incumplidas del actual enfoque de la diversidad.

Además, si analizamos los documentos de gestión en Barcelona citados en la introducción es fácil constatar que cuando se habla de diversidad en el contexto de las migraciones, en realidad se está hablando de diversidad cultural. Como establece Dietz: "la noción antropológica de diversidad cultural está transitando últimamente en las ciencias sociales y sus traducciones hacia políticas públicas desde su abierta estigmatización como "problema" — de escasa integración y/o articulación, según una noción esencialista y funcionalista de cultura-, pasando por su reivindicación como un "derecho" - de las minorías, de los pueblos indios o incluso de la humanidad entera, como en el caso de la Declaración Universal sobre Diversidad Cultural" (UNESCO 2002) —, hasta su proclamación antropológico-pedagógica como un "recurso" para la educación intercultural, para la gestión de la diversidad, para desarrollar competencias-clave en la sociedad del conocimiento" (Dietz 2011: 5). Sucede además con frecuencia que este tipo de documentos no ofrece definiciones claras acerca de qué se entiende en cada caso por cultura, algo que resulta cuanto menos llamativo dado que la diferencia cultural tiene un peso central en la justificación de gran número de planes y regulaciones. Para aquellas personas que son clasificadas como inmigrantes, la cultura se esgrime como el elemento clave de diferenciación, a menudo equiparando su cultura-etnicidad con su nacionalidad, país de nacimiento o de ascendencia, reduccionismo conocido en las ciencias sociales como nacionalismo metodológico y cuyos peligros son de sobra conocidos (Wimmer y Glick 2003). Resulta entonces paradigmático que las sustentaciones teóricas de las políticas de gestión de la diversidad cultural provean de tan poca información con relación al concepto de cultura que manejan y que además se encuentren tan alejadas de los debates conceptuales existentes en el mundo académico en torno al término y especialmente dentro de la disciplina antropológica.

Es cuanto menos sorprendente que ni en las treinta y cinco páginas del Plan Municipal para la Interculturalidad de 1997 ni en las ochenta del Plan Barcelona Interculturalidad de 2010 se defina qué es cultura. Esta ausencia es aún más elocuente si atendemos a los largos y aún irresolutos debates conceptuales (en especial dentro de la antropología) y a la variedad de acepciones del término ${ }^{14}$. Aún en ausencia de una formulación explícita en muchas de las propuestas políticas de gestión de la di-

\footnotetext{
${ }^{14}$ En un artículo repetidamente citado los antropólogos Kroeber y Kluckhohn (1952) recogían más de 150 acepciones del concepto de cultura existentes hasta aquel momento.
} 
versidad cultural (y concretamente en el planteamiento vigente de las políticas interculturales en Barcelona) es fácil percibir cómo estos enfoques utilizan una aproximación al concepto de cultura que es de naturaleza eminentemente ideacional, y que falla al poner de manifiesto el necesario engarce de esta visión inmaterial con aspectos materiales y estructurales, así como la naturaleza y dirección de tal engarce.

Resulta interesante ver cómo en el amplio proceso participativo que precedió a la elaboración del Plan Barcelona Interculturalidad los factores identificados como aquellos que dificultan las relaciones de convivencia entre los habitantes de Barcelona de orígenes culturales diversos son en gran medida subjetivos-relacionales, del tipo desconocimiento del otro, estereotipos y prejuicios, costumbres diferentes, cierre hacia el otro, etc. (Ayuntamiento de Barcelona 2010: 26), muy por encima de factores de tipo estructural-económico. Sin embargo, como establece Goldberg (1993: 128), "los cambios en los estereotipos serán más efectivos cuanto más convengan al interés de quienes sostienen los estereotipos". Es decir, es el "interés, más que el conocimiento de particularidades discordantes con el modelo [...] la fuerza transformadora de los estereotipos" (Aramburu 2002: 261). Este elemento explicaría los inesperados resultados de evaluaciones preliminares de la estrategia antirrumores, una estrategia en principio incuestionablemente exitosa y exportada como "medida estrella para hacer frente a los estereotipos y el racismo" (Moreno, Fernández y Fouassier 2016: 13) a varias ciudades españolas de la Red Española de Ciudades Interculturales. En una evaluación de la estrategia en un barrio de Bilbao los datos (sobre una muestra muy limitada eso sí) sugieren que "el impacto a corto plazo de las estrategias antirrumores son cuanto menos muy limitadas o prácticamente inexistentes y que incluso otros factores o determinantes pueden tener a corto plazo mucha más influencia que un programa de estas características" (Moreno, Fernández y Fouassier 2016: 12). En comparación con los aspectos de estructura social y exclusión, aludir al componente cultural sale barato cuando la cultura se entiende sin conexión con aspectos materiales. Como plantea Carlos Giménez (2015) en realidad la mejor política de interculturalidad sería una política de igualdad, lo que implicaría repensar la tendencia a separar la aludida diversidad cultural de otras formas de diversidad social, cuestión que a su vez complejizaría la supuesta homogeneidad hacia el interior de cada una de las culturas en contacto ya que en general se asume uniformidad intracultural frente a marcadas diferencias interculturales.

La ausencia de una concepción clara y explícita de cultura en muchos trabajos sobre diversidad cultural o inter/multi culturalidad genera un riesgo claro de reduccionismo: cultura ha sido en ocasiones igualada a expresiones artísticas y más habitualmente se reduce cultura a lenguaje y religión. Para Charles Taylor, uno de los ideólogos más conocidos del multiculturalismo, el lenguaje es el logro cultural paradigmático de la humanidad (mencionado en Benhabib 2002: 55). En la misma dirección, en Canadá por ejemplo, entre los casos de acomodo razonable de la diferencia destacan los ajustes razonables por motivos que se definen como religiosos. También en el caso barcelonés la lengua y la religión aparecen como ejes centrales (ver la cita de Joan Clos extraída del Plan de Inmigración de Barcelona del 2002 al inicio de este texto), lo que es necesario relacionar por un lado con la importancia de la lengua en la definición del denominado nacionalismo cívico catalán (Clua 2011: 65), y por otro con el papel del catolicismo en la construcción del nosotros, tanto en España como en Cataluña. 
En segundo lugar, la relación entre cultura e identidad no ha sido suficientemente problematizada, ejecutándose una traslación aproblemática de lo cultural en el individuo. "Cultura se ha vuelto un sinónimo ubicuo de identidad, un indicador y diferenciador de la identidad" (Benhabib 2002: 22). Para pasar de lo social a lo personal se ha procedido en demasiadas ocasiones de manera no suficientemente reflexiva. Sin embargo, en las actuales ciudades la mayor parte de las identidades individuales se definen a través de múltiples afinidades colectivas, ya que las personas forman parte de más de una comunidad, grupo lingüístico o ethnos (Benhabib 2002: 33). El habitante urbano está constantemente activando procesos que podríamos denominar de "travestismo identitario" (Delgado 2007: 253).

Así pues en este segundo axioma encontramos que se trabaja con definiciones simplificadas de la diversidad, en especial reducidas a ciertas visiones de la denominada diversidad cultural, que buscan gestionar a partir de lógicas empresariales la presencia de aquellos que son definidos como culturalmente diferentes, no con el fin de reducir las diferencias y desigualdades estructurales que contextualizan tal existencia, sino en aras de una aparente, por estética, paz social.

\subsection{LA INTERACCIÓN ENTRE PERSONAS DE DISTINTAS CULTURAS ES BUENA}

El principio básico del enfoque intercultural recogido en el Plan Barcelona Intercultural 2010 es que la interacción en clave positiva de personas de distintas culturas o con diferente ascendencia cultural es buena. El concepto de «interacción positiva" aparece de manera explícita por primera vez en Barcelona en el Plan de Trabajo de Inmigración 2008-2010, para pasar a convertirse en uno de los ejes centrales del Plan Barcelona Interculturalidad 2010. Este principio que se afirma de manera axiomática se sustenta sobre elementos que son en realidad problemáticos.

En primer lugar, es discutible la idea de que el contacto, sea del tipo que sea siempre que se realice en clave positiva (y este término es suficientemente ambiguo), dará lugar a fuerzas centrípetas de atracción entre las partes involucradas. Por un lado la vida de barrio, las aceras animadas, las personas que salen a los parques a tomar el fresco o el sol, son mencionadas como razones para permanecer en el barrio del Carmelo, para estar a gusto en él, incluso para seleccionarlo como lugar de residencia:

— ¿Y por qué vienes a vivir al Carmelo?

-Bueno... yo vivo con mi madre, mi madre ha decidido mudarse aquí. Cuando llegamos, vimos que era un barrio... estable. Y que se podía vivir. Y como hay más como de pueblo, que hay mucha gente, se puede hablar con la gente y que todo está tranquilo, pues, elegimos este sitio [...] Y claro, a la hora de decidir, digo, vamos a buscar un sitio, si no es un pueblo, vamos a buscar un sitio cerca de Barcelona donde podamos sentirnos como, como, como población, o como pueblo. Y ese era el sitio que encontramos que más nos gustó (Vecino reciente en el barrio, 24 años, extracomunitario, entrevista personal, 8 jun. 2016).

Por el contrario una vida de acera demasiado intensa, con mucha interacción, puede también generar reacciones negativas, como es el caso de la siguiente vecina del Carmelo: 
[...] hace ya tiempo que estoy mirando para cambiarme del barrio, ya es como... No puedo trabajar y vivir en el mismo sitio, porque es que... la gente te cansa mucho. Tú mira, yo por ejemplo, yo puedo estar paseando por la calle y yo soy de hablar un montón, conozco a mucha gente de aquí de toda la vida, aparte de clientes. Que puedo estar tomándome algo un sábado a las 11 de la noche y seguro que se acerca alguien a preguntarme por las pipetas del perro y dices, la primera vez, contestas bien; la segunda te quedas con cara de "a ver", ¿sabes? (Vecina de 38 años de toda la vida del barrio con una breve experiencia en el pueblo originario de sus padres en Extremadura, entrevista personal, 28 jun. 2016).

Gregory Bateson (1935), atendiendo a la manera concreta que adopta la interacción, identificó casos de cismogénesis o espirales crecientes de diferenciación entre individuos y grupos. La cismogénesis o progresiva diferenciación en las normas de comportamiento individual que resultan de la interacción acumulativa entre individuos (Ibid. 1935: 198), muestra cómo de hecho el contacto puede generar fuerzas centrífugas. En el caso concreto de las grandes ciudades como Barcelona, demasiada interacción traducida en una vida de barrio demasiado intensa, puede generar efectos negativos como Jane Jacobs menciona: "Cercanía es una expresión adecuadamente nauseabunda para un viejo ideal de la teoría urbanística. Este ideal es que si las personas comparten algo deben compartir muchas cosas [...] la "cercanía" resulta destructiva en las ciudades. La obligación de compartir demasiadas cosas distancia a la gente" (Jacobs 2012: 90). Estar obligados a interaccionar mucho, sobre todo entre personas que pueden no tener perfiles iguales (en cuestión de edad, costumbres, rutinas, intereses, etc.), puede llevar a que se evite la interacción como ocurre en el caso de la vecina entrevistada más arriba. La especificidad del perfil personal viene dada por cuestiones de género y del ciclo de vida, por la edad y su concreción en las capacidades y apetencias corporales, por la situación laboral que afecta a elementos como el horario, o por cuestiones relacionadas con las características de la vivienda (si se comparte, si tiene elementos exteriores, el tamaño, etc.), entre otros muchos elementos.

En el caso concreto del Carmelo además, la orografía moldea fuertemente la distribución urbanística. El barrio fue construido en las faldas de una pequeña montaña por migrantes procedentes de otras partes de España, y en especial de Andalucía, especialmente en las décadas de 1950 y 1960 en procesos de autoconstrucción ${ }^{15}$ que se tradujeron en una muy alta densidad poblacional ${ }^{16}$ junto a una baja calidad de las construcciones y un urbanismo desordenado. El resultado: paredes muy estrechas que no aíslan de los ruidos y los olores de otros pisos, zonas comunes oscuras y pequeñas, poca luz en algunas viviendas bajas; o en el caso de los espacios abiertos del barrio, aceras muy estrechas, en algunos casos insuficientes para el paso de dos personas, o plazas rodeadas de edificios donde el sonido reverbera. Este contexto constituye el espacio físico que enmarca las interacciones vecinales, y que sin lugar a dudas influye en la percibida intensidad de las mismas.

${ }^{15}$ El acceso a una vivienda digna y a servicios básicos han sido demandas tradicionales de quienes habitan el barrio. La lucha vecinal consiguió, tras años de presión y fuerte organización, la reubicación de los denominados barraquistas (chabolistas) en distintas zonas de la ciudad (Bou y Gimeno 2007).

${ }^{16} \mathrm{La}$ densidad en el Carmelo, 33,8 hab $/ \mathrm{km}^{2}$, dobla a la media de la ciudad, 15,8 hab/ $\mathrm{km}^{2}$ (Ayuntamiento de Barcelona 2016c). 
El interculturalismo busca remover las barreras a la interacción. Como ya se ha mencionado (y criticado) la estrategia antirumores encaminada a desmontar estereotipos se basa en la idea de que el desconocimiento del otro es una barrera para el acercamiento positivo. Sin embargo, las condiciones de la interacción se limitan en ocasiones a la generación de espacios específicos para encuentros puntuales. Esta interacción institucionalmente promovida tiene lugar en alguno de los espacios físicos o sociales existentes sujetos a control. De esta manera la interacción escapa de la calle en una suerte de artificialidad que no puede reproducirse en el día a día fuera de los espacios creados para tal fin. El Ayuntamiento de Barcelona en temas de fomento de las interacciones desde un enfoque intercultural aplica un claro tutelaje pedagógico sobre ciertos sectores de la población de la ciudad. El interculturalismo (es decir, el enfoque ideológico de acción pública) al buscar incidir en la interculturalidad (aspecto social de la vida en conjunto en la ciudad) puede llegar a afectarla de manera negativa debido a un exceso de injerencia, obviando los ineludibles conflictos que no pueden no existir en toda interacción o convivencia que se da en el marco de profundas desigualdades estructurales. La incorporación del conflicto en las relaciones está llevando a redefinir en el nuevo plan de interculturalidad para la ciudad de Barcelona (en elaboración en el momento de escritura de este texto) pasando de poner el énfasis en la "interacción positiva" para enfocarse en la creación de tramas relacionales (Ayuntamiento de Barcelona 2016a).

Por último, dentro del análisis de lo que he denominado el tercer axioma de la gestión intercultural de la diversidad en Barcelona, es necesario preguntarse por el énfasis en la interacción intercultural en oposición a otros tipos de interacciones, como por ejemplo intergénero o intergeneracional. Las observaciones no intrusivas llevadas a cabo en la Plaza Pastrana mostraron cómo la distribución espacial de las personas mostraba patrones de concentración sobre todo en función del género, mucho más que a partir de la racialización de los cuerpos. Los cuerpos marcados como hombre o mujer interaccionaban menos frecuentemente que los cuerpos racializados y no racializados. En especial las mujeres con niños y niñas de edades similares interaccionaban en mayor proporción que los adultos varones. Cabría preguntarse el porqué del énfasis en ciertos inter, en especial el que se relaciona con la cultura entendida en el espacio público como cuerpos con distintos grados de racialización, y la completa ausencia de interés en otro tipo de relaciones inter, en especial entre distintos géneros, generacionales y, sobre todo, estatus económico.

\section{REFLEXIONES FINALES}

El interculturalismo como ideología política guía las actuaciones públicas de tutelaje pedagógico inscritas en los planes de interculturalidad de la ciudad de Barcelona. Estas iniciativas buscan eliminar las barreras a la interacción significativa entre "diferentes culturales". Sin embargo, el aspecto material-estructural de esos obstáculos no es tenido suficientemente en cuenta, enfatizándose cuestiones subjetivo-emocionales que entienden que el desconocimiento se encuentra en la base de recelos y otros impedimentos a la interacción positiva. El énfasis en la interacción es también cuestionable, algo de lo que las propias autoridades barcelonesas actuales son conscientes 
(Ayuntamiento de Barcelona 2016a). En general resulta problemático el hecho de que la mezcla social a través de la interacción se promueva solo entre ciertos sectores sociales. La mezcla socioeconómica (a través de la nivelación de desigualdades excesivas), que sería el objeto de las denominadas políticas de igualdad, pierde visibilidad en la denominada apuesta intercultural de la ciudad.

En Barcelona la diferencia relevante en la actualidad se encarna en el inmigrante internacional, en especial aquel de niveles económicos bajos proveniente de países del Sur Global. Esta diferencia se construye en relaciones de poder específicas. Con la figura por excelencia del otro cultural, el inmigrante, no enfrentamos una definición aséptica, sino política en tanto actúa como operador cognitivo. Es por tanto ineludible poner el foco, no solo en las diferencias constitutivas de esa celebrada diversidad (cultural), sino en los subyacentes procesos de diferenciación. Más allá del significado o el contenido de las diferencias, aporta más información preguntarse por el para qué de los procesos de diferenciación. Es decir poner nuestra lente analítica, no solo en el contenido de la supuesta diferencia, sino también en la motivación que anima el proceso y el resultado que se persigue. Es por tanto, el deseo de diferenciar, y no tanto cómo se sustantive la diferencia, el aspecto relevante.

La ciudad capitalista genera diferencias que se alimentan de, a la vez que alimentan, una profunda desigualdad. Las visiones desconflictivizadas de la diversidad (cultural) que abogan por su gestión en términos de lógicas neoliberales necesitan dar paso a concepciones que incluyan el conflicto como parte integrante de toda interacción, en especial cuando esta tiene lugar en contextos de asimetría y desigualdad, como es el caso en grandes ciudades como Barcelona. Es necesario romper el paralelismo que existe entre la interacción en el modelo intercultural y el intercambio libre en el mercado de la concepción neoliberal de la economía. En ambos casos sujetos aparentemente libres y en igualdad de condiciones aceptan bien interaccionar, bien intercambiar. Quedan en la sombra las condiciones estructurales que mostrarían las asimetrías entre las partes involucradas y que afectan a la supuesta libertad de cada una de ellas.

\section{BIBLIOGRAFÍA CITADA}

Aramburu, Mikel. 2002. Los otros y nosotros. Imágenes del inmigrante en Ciutat Vella de Barcelona. Madrid: Ministerio de Educación, Cultura y Deporte.

Aramburu, Mikel. 2016. “¿Vindicando al charnego? El discurso autobiográfico de Javier Pérez Andújar y Jorge Javier Vázquez". Revista de Dialectología y Tradiciones Populares 71(1): 129-149. doi: <https://doi.org/10.3989/rdtp.2016.01.004>.

Aramburu, Mikel. 2017. "Hacerse autóctono. Cómo la inmigración del Sur de España en Cataluña se nativizó". Presentación en el XIV Congreso FAAEE-Federación de Asociaciones de Antropología del Estado Español, Valencia, 5-8 Septiembre.

Arendt, Hannah. 2003. Eichmann en Jerusalén: un estudio sobre la banalidad del mal. Barcelona: Lumen.

Ayuntamiento de Barcelona. 1997. Un Plan Municipal para la Interculturalidad. Barcelona: Ayuntamiento de Barcelona.

Ayuntamiento de Barcelona. 2002. Plan Municipal de Inmigración de Barcelona. Barcelona: Ayuntamiento de Barcelona.

Ayuntamiento de Barcelona. 2006. Ordenanza de Medidas para Fomentar y Garantizar la Convivencia Ciudadana en el Espacio Público de Barcelona. Barcelona: Ayuntamiento de Barcelona. 
Ayuntamiento de Barcelona. 2008. Plan de Trabajo Inmigración 2008-2011. Barcelona: Ayuntamiento de Barcelona.

Ayuntamiento de Barcelona. 2010. Plan Barcelona Interculturalidad. Barcelona: Ayuntamiento de Barcelona.

Ayuntamiento de Barcelona. 2012. Plan de Trabajo de Inmigración 2012-2015. Barcelona: Ayuntamiento de Barcelona.

Ayuntamiento de Barcelona. 2016a. Informe sobre los Retos y Oportunidades del Programa Bcn Interculturalidad. Barcelona: Ayuntamiento de Barcelona.

Ayuntamiento de Barcelona. 2016b. Distribución Territorial de la Renta Familiar Disponible per càpita en Barcelona. Barcelona: Departamento de Estadística.

Ayuntamiento de Barcelona. 2016c. Barcelona en Cifras 2016. Barcelona: Departamento de Estadística.

Bateson, Gregory. 1935. "Cultural Contact and Schismogenesis". Man 35: 178-183.

Benhabib, Seyla. 2002. The Claims of Culture. Equality and Diversity in the Global Era. Oxford: Princeton University Press.

Berrey, Ellen. 2014. "Breaking Glass Ceilings, Ignoring Dirty Floors: The Culture and Class Bias of Corporate Diversity Management". American Behavioral Scientist 59(2): 347-370. doi: <https:// doi.org/10.1177/0002764213503333>.

Boccagni, Paolo. 2015. "The Difference Diversity Makes: A Principle, a Lens, an Empirical Attribute for Majority-Minority Relations", en Tatiana Matejskova y Marco Antonsich (eds.), Governing through Diversity: Migration Societies in Post-multiculturalist Times: 21-38. Basingstoke: Palgrave.

Boj, Imma. 2004. "El Pabellón de las misiones: la represión de la inmigración en la Catalunya franquista”. Presentado en el 4. Congreso sobre la Inmigración en España. Girona, 10-13 Noviembre.

Bou, Lluís y Eva Gimeno. 2007. El Carmelo Ignorado. Historia de un Barrio Imposible. Barcelona: Ayuntamiento de Barcelona.

Caglar, Ayse y Nina Glick Schiller. 2015. "A Multiscalar Perspective on Cities and Migration". Sociologica 2: 1-9. doi: <https://doi.org/10.2383/81432>.

Clua, Montserrat. 2011. "Catalanes, inmigrantes y charnegos: "raza", "cultura" y "mezcla" en el discurso nacionalista catalán". Revista de Antropología Social 20: 55-75. doi: <https://doi.org/10.5209/ rev_RASO.2011.v20.36262>.

Delgado, Manuel. 2003. "¿Quién puede ser inmigrante en la ciudad?", en Manuel Delgado, Actis Walter, Danilo Martucelli, Ismael Palacín y Pedro Sáez (eds.), Exclusión Social y Diversidad Cultural: 9-24. Donostia-San Sebastián: Mugak y SOS Arrazakeria.

Delgado, Manuel. 2007. Sociedades Movedizas. Pasos hacia una Antropología de las Calles. Barcelona: Anagrama.

Delgado, Manuel. 2016. "Explotados contra excluidos. Reflexión sobre quillos, chonis y quinquis (Besòs, octubre 1990)". Presentado en el curso La Politica desde las Periferias, Cooperativa La Hidra, Barcelona, 11 Marzo.

Dietz, Gunther. 2011. "Hacia una etnografía doblemente reflexiva: Una propuesta desde la Antropología de la Interculturalidad". AIBR, Revista de Antropología Iberoamericana 6(1): 9-32.

Faist, Thomas. 2009. "Diversity: A New Mode of Incorporation?". Ethnic and Racial Studies 32(1): 171-190. doi: <https://doi.org/10.1080/01419870802483650>.

FISI. 2016. Informe sobre la Situación de la Integración de los Inmigrantes y Refugiados en España 2015. Madrid: Foro para la Integración Social de los Inmigrantes, FISI.

García, Laura. 2005. El Carmel, Ferida Oberta. De l'Esvoranc al 3 \%. Barcelona: Mina.

Giménez, Carlos. 2003. "Pluralismo, multiculturalismo e interculturalidad. Propuesta de clarificación y apuntes educativos". Educación y Futuro 8: 9-26.

Giménez, Carlos. 2015. "La Ciutadania Intercultural". Presentación en la Jornada Com Treballem la Diversitat Cultural als Districtes? Espai Avinyó, Barcelona, 3 Febrero.

Goldberg, David Theo. 1993. Racist Culture. Philosophy and the Politics of Meaning. Oxford: Blackwell.

Jacobs, Jane. 2012. Muerte y vida de las grandes ciudades. Madrid: Capitán Swing.

Kroeber, Alfred y Clyde Kluckhohn. 1952. Culture: A Critical Review of Concepts and Definitions. Papers of the Peabody Museum of American Archeology and Ethnology 47, 1. Cambridge: The Museum. 
Lavanchy, Anne, Anahí Gajardo y Fred Dervin. 2011. "Interculturality at Stake», en Anne Lavanchy, Anahí Gajardo y Fred Dervin (eds.), Politics of Interculturality: 1-24. Cambridge: Cambridge Scholars Press.

Lentin, Alana y Gavan Titley. 2008. "More Benetton than Barricades?", en Gavan Titley y Alana Lentin (eds.), The Politics of diversity in Europe: 9-27. Estrasburgo: Consejo de Europa.

Lurbe, Katia y Enrique Santamaría. 2007. "Entre (nos)otros... o la necesidad de re-pensar la construcción de las alteridades en contextos migratorios". Papers 85: 57-69. doi: <https://doi.org/ 10.5565/rev/papers.2011>.

Mata Codesal, Diana. 2016. "Es necesario desmigrantizar nuestras investigaciones?”. Ankulegi, Revista de Antropología Social 20: 47-60.

Moreno, Gorka, Iraide Fernández y Maite Fouassier. 2016. “La evaluación del proyecto antirumores de Bilbao. Estrategias metodológicas para la medición del impacto de un programa de intervención comunitaria para el fomento de la diversidad", en Domingo Carbonero, Esther Raya, Neus Caparros y Chabier Gimeno (eds.), Respuestas transdiciplinares en una sociedad global. Aportaciones desde el Trabajo Social: 1-14. Logroño: Universidad de La Rioja.

OIM. 2015. Los Migrantes y las ciudades. Nuevas colaboraciones para gestionar la movilidad. Ginebra: Organización Internacional de las Migraciones, OIM.

Pétonnet, Colette. 1982. "L'Observation flottante. L'exemple d'un cimetière parisien". L'Homme 22(4): 37-47.

Pouillon, Jean. 1998. "Appartenance et identité". Le Genre Humain 2: 189-198.

Ramírez, Eugenia. 2011. Etnicidad, Identidad, Interculturalidad: Teorías, Conceptos y Procesos de la Relacionalidad Grupal Humana. Madrid: Ed. Universitaria Ramón Areces.

UNESCO. 2002. Declaración Universal de la UNESCO sobre la Diversidad Cultural. Perú: UNESCO.

Vassallo, Brigitte. 2018. "Allò popular, allò divers, allò 'charnego'. El Crític 12 feb. Disponible en: <https://www.elcritic.cat/blogs/sentitcritic/2018/02/12/allo-popular-allo-divers-allo-charnego/>. Fecha de acceso: 14 mar. 2018.

Vertovec, Steve. 2007. "Super-Diversity and Its Implications". Ethnic and Racial Studies 30(6): 10241054. doi: <https://doi.org/10.1080/01419870701599465>.

Vidal, Tomás. 1997. "Ciutat i immigració: dos fets inseparables. El cas barceloní (segles XIX i XX)", en Manuel Delgado (ed.), Ciutat i Inmigració: 17-37. Barcelona: Centre Cultura Comtemporània de Barcelona.

White, Bob. 2013. "Contre la diversité". TicArtToc (Réflexions): 44-47.

Wimmer, Andreas y Nina Glick Schiller. 2003. "Methodological nationalism and beyond: nation-state building, migration and the social sciences". Global Networks 2(4): 301-334. doi: <https://doi.org/ 10.1111/1471-0374.00043>.

Fecha de recepción: 5 de julio de 2017

Fecha de aceptación: 16 de febrero de 2018 